\title{
Relations for Single and Product Moments of Odds Generalized Exponential-Pareto Distribution Based on Generalized Order Statistics and Characterization
}

\author{
Neetu Gupta ${ }^{1, *}$, Zaki Anwar ${ }^{1}$ \\ ${ }^{1}$ Department of Statistics and Operations Research, Aligarh Muslim University, India.
}

\begin{abstract}
This paper deals with explicit expressions and recurrence relations for single, inverse, product and ratio moments of Generalized Order Statistics from Odds Generalized Exponential-Pareto Distribution (OGEPD). Characterization results have also been carried out.
\end{abstract}

Keywords Generalized order statistics, order statistics, record values, recurrence relations.

AMS 2010 subject classifications 62G30, 62E10, 60E05

DOI: $10.19139 /$ soic.v7i1.478

\section{Introduction}

The concept of generalized order statistics ( $g o s)$ was first introduced by [8] which envelops variety of models of ordered random variables that acts as a flexible model in various directions such as order statistics, upper record values, progressive type II censoring order statistics, sequential order statistics and Pfeiffer's records.

Let $X_{1}, X_{2}, \ldots$ be a sequence of independent identically distributed (iid) random variables with distribution function (df) $F(x)$ and probability density function $(p d f) f(x)$. Let $k \geq 1, n \geq 2, n \in N, \tilde{m}=\left(m_{1}, m_{2}, \ldots, m_{n-1}\right) \in$ $\Re^{n-1}, M_{r}=\sum_{j=r}^{n-1} m_{j}$ such that $\gamma_{r}=k+n-r+M_{r}>0 \forall r \in\{1,2, \ldots, n-1\}$. Then $X(r, n, \tilde{m}, k), r=$ $1,2, \ldots, n$, is called gos based on $F(x)$, if their joint $p d f$ is of the form

$k\left(\prod_{j=1}^{n-1} \gamma_{j}\right)\left(\prod_{i=1}^{n-1}\left[1-F\left(x_{i}\right)\right]^{m_{i}} f\left(x_{i}\right)\right)\left[1-F\left(x_{n}\right)\right]^{k-1} f\left(x_{n}\right), \quad F^{-1}(0)<x_{1} \leq \ldots \leq x_{n}<F^{-1}(1)$

For different values of $m_{i}$ 's, $k$ and $\gamma_{i}$ 's the model of gos reduces to various models e.g., when $\left(m_{1}=m_{2}=\ldots=m_{n-1}=0, k=1, \gamma_{i}=1+n-i\right)$, this model reduces to order statistics and for $k^{t h}$ upper record values $\left(m_{1}=m_{2}=\ldots=m_{n-1}=-1\right.$, i.e. $\left.\gamma_{i}=k, k \in N\right)$. Here we take the case $m_{i}=m_{j}=m$. Then the density function of $r^{t h} \operatorname{gos} X(r, n, m, k)$ is given by

$$
f_{X(r, n, m, k)}=\frac{C_{r-1}}{(r-1) !}[\bar{F}(x)]^{\gamma_{r}-1} f(x) g_{m}^{r-1}(F(x))
$$

\footnotetext{
*Correspondence to: Neetu Gupta (Email: ngb5433@gmail.com). Department of Statistics and Operations Research, Aligarh Muslim University, Aligarh, India (202002).
}

ISSN 2310-5070 (online) ISSN 2311-004X (print)

Copyright (C) 2019 International Academic Press 
The joint $p d f$ of $X(r, n, m, k)$ and $X(s, n, m, k), 1 \leq r<s \leq n$ is

$$
\begin{array}{r}
f_{X(r, n, m, k) X(s, n, m, k)}=\frac{C_{s-1}}{(r-1) !(s-r-1) !}[\bar{F}(x)]^{m} f(x) g_{m}^{r-1}(F(x))\left[h_{m} F(y)-h_{m} F(x)\right]^{s-r-1} \\
\times[\bar{F}(y)]^{\gamma_{s}-1} f(y),
\end{array}
$$

where

$$
\begin{gathered}
\bar{F}(x)=1-F(x), C_{r-1}=\prod_{i=1}^{r} \gamma_{i}, \\
h_{m}(x)=\left\{\begin{array}{lc}
-\frac{1}{m+1}(1-x)^{m+1}, & m \neq-1 \\
-\ln (1-x), & m=-1
\end{array}\right.
\end{gathered}
$$

and

$$
g_{m}(x)=h_{m}(x)-h_{m}(0), x \in[0,1) .
$$

A lot of work has been carried out by several researchers in the field of gos. Recurrence relations for moments of gos for specific as well as for general class of distribution have been well investigated hitherto. [2] and [11] derived the recurrence relations for single and product moments of gos for some general class of distributions, whereas explicit expressions for exact moments of gos were probed by [4] and the generalized record values from additive Weibull distribution derived by [13]. For more details of gos we have [9, 12, 3, 1, 14, 10] and [6]. A random variable $X$ is said to have OGEPD [15] if its $d f$ is given by

$$
F(x)=1-e^{-\lambda\left[\left(\frac{x}{a}\right)^{\theta}-1\right]}, \quad x>a, \theta, \lambda>0 .
$$

The corresponding $p d f$ is of the form

$$
f(x)=\frac{\lambda \theta}{a^{\theta}} x^{\theta-1} e^{-\lambda\left[\left(\frac{x}{a}\right)^{\theta}-1\right]}, \quad x>a, \theta, \lambda>0 .
$$

The relation between $\bar{F}(x)$ and $f(x)$ can be easily derived as

$$
\bar{F}(x)=\frac{a^{\theta}}{\lambda \theta} x^{1-\theta} f(x),
$$

where $\bar{F}(x)=1-F(x)$.

\section{Relation for single moments}

\subsection{Explicit expressions for single moments}

We first derive explicit expression for single moments of $r^{t h}$ gos, $X(r, n, m, k)$. The following theorem shows the explicit expression for $E\left[X^{j}(r, n, m, k)\right]=\mu_{r: n}^{(j)}$.

Theorem 1

For $1 \leq r \leq n, k \geq 1$ and $j=0,1,2, \ldots$,

$$
\mu_{r: n, m, k}^{(j)}=\frac{C_{r-1}}{(r-1) !(m+1)^{r-1}} \frac{a^{j}}{\lambda^{j / \theta}} \sum_{u=0}^{r-1}(-1)^{u}\left(\begin{array}{c}
r-1 \\
u
\end{array}\right) e^{\lambda \gamma_{r-u}}\left(\gamma_{r-u}\right)^{-j / \theta-1} \Gamma\left((j / \theta+1), \lambda \gamma_{r-u}\right),
$$

and for $m=-1$, we have

$$
\mu_{r: n,-1, k}^{(j)}=\frac{e^{\lambda k} a^{j}}{(r-1) !} \sum_{u=0}^{r-1}(-1)^{r-u-1}\left(\begin{array}{c}
r-1 \\
u
\end{array}\right) \frac{\Gamma((j / \theta+u+1), \lambda k)}{(\lambda k)^{j / \theta+u-r+1}} .
$$


Proof

Using (1), we get

$$
\mu_{r: n, m, k}^{(j)}=\frac{C_{r-1}}{(r-1) !} \int_{a}^{\infty} x^{j}[\bar{F}(x)]^{\gamma_{r}-1} g_{m}^{r-1}(F(x)) f(x) d x .
$$

On expanding $g_{m}^{r-1}(F(x))=\left[\frac{1}{m+1}\left(1-(\bar{F}(x))^{m+1}\right)\right]^{r-1}$ binomially in above expression, we get

$$
\mu_{r: n, m, k}^{(j)}=\frac{C_{r-1}}{(r-1) !(m+1)^{r-1}} \sum_{u=0}^{r-1}(-1)^{u}\left(\begin{array}{c}
r-1 \\
u
\end{array}\right) \int_{a}^{\infty} x^{j}[\bar{F}(x)]^{\gamma_{r-u}-1} f(x) d x .
$$

On using (3) and (4) in (8), we obtain

$$
\begin{aligned}
& \mu_{r: n, m, k}^{(j)}=\frac{\lambda \theta}{a^{\theta}} \frac{C_{r-1}}{(r-1) !(m+1)^{r-1}} \sum_{u=0}^{r-1}(-1)^{u}\left(\begin{array}{c}
r-1 \\
u
\end{array}\right) e^{\lambda \gamma_{r-u}} \int_{a}^{\infty} x^{j+\theta-1} e^{-\frac{\lambda}{a^{\theta}} \gamma_{r-u} x^{\theta}} d x \\
& \mu_{r: n, m, k}^{(j)}=\frac{C_{r-1}}{(r-1) !(m+1)^{r-1}} \frac{\lambda \theta}{a^{\theta}} \sum_{u=0}^{r-1}(-1)^{u}\left(\begin{array}{c}
r-1 \\
u
\end{array}\right) e^{\lambda \gamma_{r-u}} \int_{a}^{\infty} x^{j+\theta-1} e^{-\frac{\lambda}{a^{\theta}} \gamma_{r-u} x^{\theta}} d x .
\end{aligned}
$$

From [5] p-346, we have

$$
\int_{u}^{\infty} x^{m} e^{-\beta x^{n}} d x=\frac{\Gamma\left((m+1) / n, \beta u^{n}\right)}{n \beta^{(m+1) / n}}, \quad \beta, m, n>0 .
$$

By substituting (10) in (9) and simplifying the resulting expression, we get the required result for $m \neq-1$.

When $m=-1$, we have

$$
\mu_{r: n,-1, k}^{(j)}=\frac{k^{r}}{(r-1) !} \int_{a}^{\infty} x^{j}[-\ln \bar{F}(x)]^{r-1}[\bar{F}(x)]^{k-1} f(x) d x .
$$

From (3) and (4), we have

$$
\begin{aligned}
\mu_{r: n,-1, k}^{(j)}= & \frac{k^{r}}{(r-1) !} \frac{\lambda^{r} \theta}{a^{\theta}} e^{\lambda k} \int_{a}^{\infty} x^{j+\theta-1}\left(\left(\frac{x}{a}\right)^{\theta}-1\right)^{r-1} e^{-\lambda k\left(\frac{x}{a}\right)^{\theta}} d x \\
\mu_{r: n,-1, k}^{j}= & \frac{(\lambda k)^{r} e^{\lambda k}}{(r-1) ! \theta} \sum_{u=0}^{r-1}(-1)^{r-u-1}\left(\begin{array}{c}
r-1 \\
u
\end{array}\right) \frac{1}{a^{\theta(u+1)}} \\
& \times \int_{a}^{\infty} x^{j+\theta(u+1)-1} e^{-\frac{\lambda k}{a^{\theta}} x^{\theta}} d x .
\end{aligned}
$$

On using (10) in (11), we get the required result for $m=-1$.

\section{Special Cases}

(i) For $m=0, k=1$ in (6), we get the explicit formula for the single moments of ordinary order statistics for the OGEPD as

$$
\begin{aligned}
\mu_{r: n}^{(j)}=C_{r: n} a^{j} \lambda^{-j / \theta} \sum_{u=0}^{r-1}(-1)^{u}\left(\begin{array}{c}
r-1 \\
u
\end{array}\right) e^{\lambda(n-r+u+1)} \Gamma((j / \theta+1), \lambda(n-r+u+1)) \\
\times(n-r+u+1)^{-(j / \theta+1)} .
\end{aligned}
$$

(ii) For $k=1$ in (7), we get the explicit expression for the single moments of upper record values for the OGEPD as

$$
\mu_{r: n,-1,1}^{(j)}=\frac{e^{\lambda} a^{j}}{(r-1) !} \sum_{u=0}^{r-1}(-1)^{r-u-1}\left(\begin{array}{c}
r-1 \\
u
\end{array}\right) \frac{\Gamma((j / \theta+u+1), \lambda)}{\lambda^{j / \theta+u-r+1}} .
$$




\subsection{Recurrence relation for single moments}

Theorem 2

If $X$ is a $r v$ with $d f(3)$ with $2 \leq r \leq n, j \geq 0, k \geq 1$ the following recurrence relation

$$
\mu_{r: n, m, k}^{(j)}=\mu_{r-1: n, m, k}^{(j)}+\frac{a^{\theta} j}{\lambda \theta \gamma_{r}} \mu_{r: n, m, k}^{(j-\theta)}, \quad m \neq-1
$$

is satisfied.

Proof

To obtain the recurrence relation for single moments of gos, we use the result of [2] for the OGEPD

$$
\mu_{r: n, m, k}^{(j)}-\mu_{r-1: n, m, k}^{(j)}=\frac{j C_{r-1}}{\gamma_{r}(r-1) !} \int_{a}^{\infty} x^{j-1}[\bar{F}(x)]^{\gamma_{r}} g_{m}^{r-1}(F(x)) d x
$$

Using (5) in (13), we get

$$
\mu_{r: n, m, k}^{(j)}=\mu_{r-1: n, m, k}^{(j)}+\frac{a^{\theta} j C_{r-1}}{\lambda \theta \gamma_{r}(r-1) !} \int_{a}^{\infty} x^{j-\theta}[\bar{F}(x)]^{\gamma_{r}-1} g_{m}^{r-1}(F(x)) f(x) d x
$$

On simplifying the above expression, we get the required result of (12).

\section{Special Cases}

(i) For $m=0, k=1$ in (12), we get the recurrence relations for single moments of ordinary order statistics for the OGEPD as

$$
\mu_{r: n}^{(j)}=\mu_{r-1: n}^{(j)}+\frac{a^{\theta} j}{\lambda \theta(n-r+1)} \mu_{r: n}^{(j-\theta)} .
$$

(i) For $m=-1, k=1$ in (12), we get the recurrence relations for single moments of upper record values for the OGEPD as

$$
\mu_{r: n,-1,1}^{(j)}=\mu_{r-1: n,-1,1}^{(j)}+\frac{a^{\theta} j}{\lambda \theta} \mu_{r: n,-1,1}^{(j-\theta)} .
$$


Table 1(a): First Four Moments of Order Statistics

\begin{tabular}{|c|c|c|c|c|c|c|c|c|c|}
\hline \multirow[b]{2}{*}{$n$} & \multirow[b]{2}{*}{$r$} & \multicolumn{4}{|c|}{$a=0.5, \lambda=1, \theta=0.5$} & \multicolumn{4}{|c|}{$a=0.5, \lambda=1.5, \theta=0.5$} \\
\hline & & $\mu^{(1)}$ & $\mu^{(2)}$ & $\mu^{(3)}$ & $\mu^{(4)}$ & $\mu^{(1)}$ & $\mu^{(2)}$ & $\mu^{(3)}$ & $\mu^{(4)}$ \\
\hline 1 & 1 & 2.50000 & 16.2500 & 244.620 & 6850.11 & 1.61110 & 5.21290 & 35.3780 & 440.660 \\
\hline \multirow[t]{2}{*}{2} & 1 & 1.25000 & 2.62500 & 10.3438 & 72.7188 & 0.94444 & 1.21296 & 2.39660 & 7.68527 \\
\hline & 2 & 3.75000 & 29.8750 & 478.906 & 13627.4 & 2.27778 & 9.21296 & 68.3596 & 873.628 \\
\hline \multirow[t]{3}{*}{3} & 1 & 0.94444 & 1.21296 & 2.39660 & 7.68527 & 0.77161 & 0.70085 & 0.81081 & 1.29473 \\
\hline & 2 & 1.86111 & 5.44907 & 26.2380 & 202.786 & 1.29012 & 2.23720 & 5.56819 & 20.4663 \\
\hline & 3 & 4.69444 & 42.0880 & 705.240 & 20339.7 & 2.77160 & 12.7008 & 99.7553 & 1300.21 \\
\hline \multirow[t]{4}{*}{4} & 1 & 0.81250 & 0.80467 & 1.06689 & 2.05457 & 0.69444 & 0.53241 & 0.47184 & 0.512817 \\
\hline & 2 & 1.34028 & 2.43778 & 6.38574 & 24.5774 & 1.00309 & 1.20616 & 1.82774 & 3.64048 \\
\hline & 3 & 2.38194 & 8.46036 & 46.0903 & 380.994 & 1.57716 & 3.26823 & 9.30864 & 37.2922 \\
\hline & 4 & 5.46528 & 53.2972 & 924.957 & 26992.6 & 3.16975 & 15.8451 & 129.904 & 1721.18 \\
\hline \multirow[t]{5}{*}{5} & 1 & 0.74000 & 0.62760 & 0.65156 & 0.89225 & 0.65111 & 0.45279 & 0.34574 & 0.30127 \\
\hline & 2 & 1.10250 & 1.51304 & 2.72823 & 6.70384 & 0.86778 & 0.85089 & 0.97621 & 1.35901 \\
\hline & 3 & 1.69694 & 3.82492 & 11.872 & 51.3877 & 1.20605 & 1.73906 & 3.10503 & 7.0627 \\
\hline & 4 & 2.83861 & 11.5507 & 68.9026 & 600.732 & 1.82457 & 4.28768 & 13.4444 & 57.4452 \\
\hline & 5 & 6.12194 & 63.7338 & 1138.97 & 33590.6 & 3.50605 & 18.7344 & 159.019 & 2137.11 \\
\hline & & \multicolumn{4}{|c|}{$a=1, \lambda=2, \theta=1$} & \multicolumn{4}{|c|}{$a=1.5, \lambda=2, \theta=2$} \\
\hline 1 & 1 & 1.50000 & 2.50000 & 4.75000 & 10.5000 & 1.81600 & 3.37500 & 6.43945 & 12.6556 \\
\hline \multirow[t]{2}{*}{2} & 1 & 1.25000 & 1.62500 & 2.21875 & 3.21875 & 1.66975 & 2.81250 & 4.78385 & 8.22656 \\
\hline & 2 & 1.75000 & 3.37500 & 7.28125 & 17.7813 & 1.96230 & 3.93750 & 8.09524 & 17.0859 \\
\hline \multirow[t]{3}{*}{3} & 1 & 1.16667 & 1.38889 & 1.69444 & 2.12963 & 1.61648 & 2.62500 & 4.28427 & 7.03125 \\
\hline & 2 & 1.41667 & 2.09722 & 3.26736 & 5.39699 & 1.77631 & 3.18750 & 5.78303 & 10.6172 \\
\hline & 3 & 1.91667 & 4.01389 & 9.28819 & 23.9734 & 1.58874 & 2.53125 & 4.04525 & 6.48633 \\
\hline \multirow[t]{4}{*}{4} & 1 & 1.12500 & 1.28125 & 1.48047 & 1.74023 & 1.69968 & 2.90625 & 5.00132 & 8.66602 \\
\hline & 2 & 1.29167 & 1.71181 & 2.33637 & 3.29782 & 1.69968 & 2.90625 & 5.00132 & 8.66602 \\
\hline & 3 & 1.54167 & 2.48264 & 4.19835 & 7.49617 & 1.85293 & 3.46875 & 6.56473 & 12.5684 \\
\hline & 4 & 2.04167 & 4.52431 & 10.9848 & 29.4658 & 2.12275 & 4.59375 & 10.1469 & 22.9043 \\
\hline \multirow[t]{5}{*}{5} & 1 & 1.10000 & 1.22000 & 1.36600 & 1.54640 & 1.57171 & 2.47500 & 3.90545 & 6.17625 \\
\hline & 2 & 1.22500 & 1.52625 & 1.93834 & 2.51557 & 1.65690 & 2.75625 & 4.60445 & 7.72664 \\
\hline & 3 & 1.39167 & 1.99014 & 2.93341 & 4.47118 & 1.76385 & 3.13125 & 5.59662 & 10.0751 \\
\hline & 4 & 1.64167 & 2.81097 & 5.04164 & 9.51282 & 1.91232 & 3.69375 & 7.21014 & 14.2305 \\
\hline & 5 & 2.14167 & 4.95264 & 12.4706 & 34.4540 & 2.17536 & 4.81875 & 10.8811 & 25.0727 \\
\hline
\end{tabular}

Table 1(b): First Four Moments of Upper Record Values

\begin{tabular}{l|llll|llll}
\hline \multicolumn{5}{c}{$a=1, \lambda=1.5, \theta=1.5$} & \multicolumn{4}{c}{$a=1, \lambda=1.5, \theta=2$} \\
\hline$r$ & $\mu^{(1)}$ & $\mu^{(2)}$ & $\mu^{(3)}$ & $\mu^{(4)}$ & $\mu^{(1)}$ & $\mu^{(2)}$ & $\mu^{(3)}$ & $\mu^{(4)}$ \\
\hline 2 & 2.1815 & 2.3019 & 1.7407 & 0.9008 & 2.0469 & 2.3330 & 2.2169 & 1.7407 \\
3 & 5.3586 & 7.6724 & 8.4676 & 7.2166 & 4.7019 & 6.6249 & 8.0576 & 8.4676 \\
4 & 6.5712 & 10.015 & 11.276 & 9.1401 & 5.6509 & 8.4167 & 10.622 & 11.276 \\
5 & 8.4753 & 15.086 & 20.191 & 20.352 & 7.0222 & 11.716 & 16.667 & 20.191 \\
6 & 9.8931 & 19.427 & 28.089 & 29.546 & 7.9757 & 14.395 & 21.931 & 28.089 \\
\hline \multicolumn{3}{c}{$a=1, \lambda=0.5, \theta=2.5$} & & \multicolumn{4}{c}{$a=1, \lambda=0.5, \theta=3$} \\
\hline 2 & 1.2316 & 3.0133 & 8.7309 & 31.545 & 1.0698 & 2.2041 & 5.0000 & 13.055 \\
3 & 2.2616 & 5.1485 & 15.429 & 59.819 & 2.0309 & 3.7902 & 8.6253 & 23.565 \\
4 & 2.5384 & 6.6505 & 22.047 & 93.163 & 2.2088 & 4.703 & 11.746 & 34.691 \\
5 & 2.9423 & 8.2108 & 29.176 & 131.96 & 2.5389 & 5.6769 & 15.018 & 46.973 \\
6 & 3.2511 & 9.6893 & 36.614 & 175.62 & 2.7729 & 6.5583 & 18.283 & 60.130 \\
\hline
\end{tabular}




\section{Relations for inverse moments}

In this section, we derive recurrence relation for inverse moments of gos. The inverse moments of gos are defined as

$$
\mu_{r: n, m, k}^{(-j)}=E\left(X_{r: n, m, k}^{-j}\right)=\int_{-\infty}^{\infty} x^{-j} f_{r: n, m, k}(x) d x .
$$

Theorem 3

Fix a positive integer $k$ and for $d f$ (3) with $2 \leq r \leq n, j=0,1,2, \ldots$ the following recurrence relation

$$
\mu_{r: n, m, k}^{(-j)}=\mu_{r-1: n, m, k}^{(-j)}-\frac{j a^{\theta}}{\gamma_{r} \lambda \theta} \mu_{r: n, m, k}^{(-j-\theta)}, \quad m \neq-1
$$

is satisfied.

Proof

From (14) and (1), we have

$$
\mu_{r: n, m, k}^{(-j)}=\frac{C_{r-1}}{(r-1) !} \int_{a}^{\infty} x^{-j}[\bar{F}(x)]^{\gamma_{r-1}} f(x) g_{m}^{r-1}(F(x)) d x .
$$

Proceeding in similar manner as we have done for Theorem 2, we get

$$
\mu_{r: n, m, k}^{(-j)}=\mu_{r-1: n, m, k}^{(-j)}-\frac{j C_{r-1}}{\gamma_{r}(r-1) !} \int_{a}^{\infty} x^{-j-1}[\bar{F}(x)]^{\gamma_{r}} g_{m}^{r-1}(F(x)) d x .
$$

By using (5) in above expression, we have

$$
\mu_{r: n, m, k}^{(-j)}=\mu_{r-1: n, m, k}^{(-j)}-\frac{j C_{r-1}}{\gamma_{r}(r-1) !} \frac{a^{\theta}}{\lambda \theta} \int_{a}^{\infty} x^{-j-\theta}[\bar{F}(x)]^{\gamma_{r}-1} g_{m}^{r-1}(F(x)) f(x) d x .
$$

On simplifying (16), we get the required result of (15).

\section{Special Cases}

(i) For $m=0, k=1$ in (15), we get the recurrence relation for inverse moments of ordinary order statistics for the OGEPD as

$$
\mu_{r: n}^{(-j)}=\mu_{r-1: n}^{(-j)}-\frac{a^{\theta} j}{\lambda \theta(n-r+1)} \mu_{r: n}^{(-j-\theta)} .
$$

(ii) For $m=-1, k=1$ in (15), we get the recurrence relation for inverse moments of upper record values for the OGEPD as

$$
\mu_{r: n,-1,1}^{(-j)}=\mu_{r-1: n,-1,1}^{(-j)}-\frac{a^{\theta} j}{\lambda \theta} \mu_{r: n,-1,1}^{(-j-\theta)} .
$$

\section{Relations for product moments}

Theorem 4

For the OGEPD given in (3), $1 \leq r<s \leq n-1, k \geq 1, j \geq 0$ the following recurrence relation

$$
\mu_{r, s: n, m, k}^{(i, j)}=\mu_{r, s-1: n, m, k}^{(i, j)}+\frac{a^{\theta} j}{\lambda \theta \gamma_{s}} \mu_{r, s: n, m, k}^{(i, j-\theta)}, m \neq-1
$$

is satisfied. 
Proof

From (2), we have

$$
\begin{aligned}
\mu_{r, s: n, m, k}^{(i, j)}=\frac{C_{s-1}}{(r-1) !(s-r-1) !} \int_{-\infty}^{\infty} & \int_{x}^{\infty} x^{i} y^{j}[\bar{F}(x)]^{m} f(x) g_{m}^{r-1}(F(x))\left[h_{m} F(y)-h_{m} F(x)\right]^{s-r-1} \\
& \times[\bar{F}(y)]^{\gamma_{s}-1} f(y) d y d x .
\end{aligned}
$$

By considering the result of [2] for product moments of any distribution, we have

$$
\begin{array}{r}
\mu_{r, s: n, m, k}^{(i, j)}=\mu_{r, s-1: n, m, k}^{(i, j)}+\frac{C_{s-1} j}{\gamma_{s}(r-1) !(s-r-1) !} \int_{-\infty}^{\infty} \int_{x}^{\infty} x^{i} y^{j-1}[\bar{F}(x)]^{m} f(x) g_{m}^{r-1}(F(x)) \\
{\left[h_{m} F(y)-h_{m} F(x)\right]^{s-r-1}[\bar{F}(y)]^{\gamma_{s}} d y d x .}
\end{array}
$$

Using the relation (5) in (18) for the OGEPD, we have

$$
\begin{aligned}
\mu_{r, s: n, m, k}^{(i, j)}=\mu_{r, s-1: n, m, k}^{(i, j)}+\frac{C_{s-1} j}{\gamma_{s}(r-1) !(s-r-1) !} \frac{a^{\theta}}{\lambda \theta} \int_{a}^{\infty} \int_{x}^{\infty} x^{i} y^{j-\theta}[\bar{F}(x)]^{m} f(x) g_{m}^{r-1}(F(x)) \\
{\left[h_{m} F(y)-h_{m} F(x)\right]^{s-r-1} f(y)[\bar{F}(y)]^{\gamma_{s}-1} d y d x . }
\end{aligned}
$$

On simplifying (19), we get the result of (17).

\section{Special Cases}

(i) For $m=0, k=1$ in (17), we get the recurrence relations for product moments of ordinary order statistics for the OGEPD as

$$
\mu_{r, s: n}^{(i, j)}=\mu_{r, s-1: n}^{(i, j)}+\frac{a^{\theta} j}{\lambda \theta(n-s+1)} \mu_{r, s: n}^{(i, j-\theta)} .
$$

(ii) For $m=-1, k=1$ in (17), we get the recurrence relations for product moments of upper record values for the OGEPD as

$$
\mu_{r, s: n,-1,1}^{(i, j)}=\mu_{r, s-1: n,-1,1}^{(i, j)}+\frac{a^{\theta} j}{\lambda \theta} \mu_{r: n,-1,1}^{(i, j-\theta)} .
$$

\section{Relations for ratio moments}

The ratio moments of gos are defined as

$$
\mu_{r, s: n, m, k}^{(i,-j)}=\int_{-\infty}^{\infty} \int_{x}^{\infty} \frac{x^{i}}{y^{j}} f_{r, s: n, m, k}(x, y) d y d x=\int_{-\infty}^{\infty} \int_{x}^{\infty} x^{i} y^{-j} f_{r, s: n, m, k}(x, y) d y d x .
$$

Theorem 5

For the OGEPD given in (3), $1 \leq r<s \leq n-1, k \geq 1$ and $j \geq 0$ the following recurrence relation for ratio moments

$$
\mu_{r, s: n, m, k}^{(i,-j)}=\mu_{r, s-1: n, m, k}^{(i,-j)}-\frac{a^{\theta} j}{\lambda \theta \gamma_{s}} \mu_{r, s: n, m, k}^{(i,-j-\theta)}, \quad m \neq-1
$$

is satisfied.

Proof

We derive the ratio moments for the OGEPD by using (20) and proceeding in similar way as Theorem 4, we have

$$
\begin{aligned}
\mu_{r, s: n, m, k}^{(i,-j)}=\mu_{r, s-1: n, m, k}^{(i,-j)}-\frac{C_{s-1} j}{\gamma_{s}(r-1) !(s-r-1) !} \int_{a}^{\infty} \int_{x}^{\infty} & x^{i} y^{-j-1}[\bar{F}(x)]^{m} f(x) g_{m}^{r-1}(F(x)) \\
& \times\left[h_{m} F(y)-h_{m} F(x)\right]^{s-r-1}[\bar{F}(y)]^{\gamma_{s}} d y d x .
\end{aligned}
$$

On using (5), we get the result of (21) . 


\section{Special cases}

(i) For $m=0, k=1$ in (21), we get the recurrence relations for ratio moments of ordinary order statistics for the OGEPD as

$$
\mu_{r, s: n}^{(i,-j)}=\mu_{r, s-1: n}^{(i,-j)}-\frac{a^{\theta} j}{\lambda \theta(n-s+1)} \mu_{r, s: n}^{(i,-j-\theta)} .
$$

(ii) For $m=-1, k=1$ in (21), we get the recurrence relations for ratio moments of upper record values for the OGEPD as

$$
\mu_{r, s: n,-1,1}^{(i,-j)}=\mu_{r, s-1: n,-1,1}^{(i,-j)}-\frac{a^{\theta} j}{\lambda \theta} \mu_{r: n,-1,1}^{(i,-j-\theta)} .
$$

\section{Characterization}

\subsection{Characterization of OGEPD based on a recurrence relation for single moments}

Theorem 6

For the positive integers $k$ and $j$, a necessary and sufficient condition for a random variable $X$ to be distributed with $c d f$ given in (3) is that

$$
\mu_{r: n, m, k}^{(j)}=\mu_{r-1: n, m, k}^{(j)}+\frac{a^{\theta} j}{\lambda \theta \gamma_{r}} \mu_{r: n, m, k}^{(j-\theta)} .
$$

Proof

The necessary part follows immediately from (12). On the other hand if the recurrence relation in (22) is satisfied, then from (1), we have

$$
\begin{array}{r}
\frac{C_{r-1}}{\gamma_{r}(r-1) !} \int_{a}^{\infty} x^{j}[\bar{F}(x)]^{\gamma_{r}} g_{m}^{r-2}(F(x)) f(x)\left(\frac{\gamma_{r} g_{m}(F(x))}{\bar{F}(x)}-(r-1)[\bar{F}(x)]^{m}\right) d x=\frac{C_{r-1} j}{(r-1) ! \gamma_{r}} \frac{a^{\theta}}{\lambda \theta} \\
\times \int_{a}^{\infty} x^{j-\theta}[\bar{F}(x)]^{\gamma_{r}-1} g_{m}^{r-1}(F(x)) f(x) d x .
\end{array}
$$

Consider $\xi(x)=-g_{m}^{r-1}(F(x))[\bar{F}(x)]^{\gamma_{r}}$.

Differentiating $\xi(x)$ with respect to $x$, we have

$$
\xi^{\prime}(x)=g_{m}^{r-2}(F(x)) f(x)[\bar{F}(x)]^{\gamma_{r}}\left[\frac{g_{m}(F(x)) \gamma_{r}}{\bar{F}(x)}-(r-1)[\bar{F}(x)]^{m}\right] .
$$

Thus, we have

$$
\frac{C_{r-1}}{(r-1) ! \gamma_{r}} \int_{a}^{\infty} x^{j} \xi^{\prime}(x) d x=-\frac{C_{r-1} j}{(r-1) ! \gamma_{r}} \frac{a^{\theta}}{\lambda \theta} \int_{a}^{\infty} x^{j-\theta} \xi(x)[\bar{F}(x)]^{-1} f(x) d x .
$$

Integrating left hand side of (24) and using the expression of $\xi(x)$, we have

$$
\frac{C_{r-1}}{(r-1) ! \gamma_{r}} \int_{a}^{\infty} x^{j-1} g_{m}^{r-1}(F(x))[\bar{F}(x)]^{\gamma_{r}}\left(1-\frac{a^{\theta}}{\lambda \theta} x^{1-\theta} \frac{f(x)}{\bar{F}(x)}\right)=0 .
$$

Using generalization result of Müntz-Szász Theorem of [7], we get $\frac{\bar{F}(x)}{f(x)}=\frac{a^{\theta}}{\lambda \theta} x^{1-\theta}$ which proves that,

$$
\bar{F}(x)=\frac{a^{\theta}}{\lambda \theta} x^{1-\theta} f(x), x>a, \theta, \lambda>0 .
$$

By simplifying above expression, we can also get the following result,

$$
f(x)=\frac{\lambda \theta}{a^{\theta}} x^{\theta-1} e^{-\lambda\left[\left(\frac{x}{a}\right)^{\theta}-1\right]}, x>a, \theta, \lambda>0 .
$$




\subsection{Characterization of OGEPD based on conditional moments}

Theorem 7

If $X$ is an absolutely continuous positive random variable with $d f G(x)$ and $g(x)$ such that $E(X)$ exist, then $E(X \mid X \leq x)=h(x) \varphi(x)$ where $\varphi(x)=g(x) / G(x)$, then

$$
h(x)=\frac{a^{\theta+1}}{\lambda \theta} x^{1-\theta} e^{\lambda\left[\left(\frac{x}{a}\right)^{\theta}-1\right]}-\frac{a^{\theta}}{\lambda \theta} x^{2-\theta}+\frac{a^{\theta}}{\lambda \theta} x^{1-\theta} e^{\lambda\left[\left(\frac{x}{a}\right)^{\theta}-1\right]} \int_{a}^{x} e^{-\lambda\left[\left(\frac{u}{a}\right)^{\theta}-1\right]} d u
$$

holds if and only if

$$
g(x)=\frac{\lambda \theta}{a^{\theta}} x^{\theta-1} e^{-\lambda\left[\left(\frac{x}{a}\right)^{\theta}-1\right]}, \quad x \geq a, \theta, \lambda>0 .
$$

Proof

We first prove the necessity part. For this, we consider

$$
g(x)=\frac{\lambda \theta}{a^{\theta}} x^{\theta-1} e^{-\lambda\left[\left(\frac{x}{a}\right)^{\theta}-1\right]},
$$

then, we take

$$
h(x)=\frac{1}{g(x)} \int_{a}^{x} u f(u) d u .
$$

On simplifying (26) and using $g(x)$, we get

$$
h(x)=\frac{a^{\theta+1}}{\lambda \theta} x^{1-\theta} e^{\lambda\left[\left(\frac{x}{a}\right)^{\theta}-1\right]}-\frac{a^{\theta}}{\lambda \theta} x^{2-\theta}+\frac{a^{\theta}}{\lambda \theta} x^{1-\theta} e^{\lambda\left[\left(\frac{x}{a}\right)^{\theta}-1\right]} \int_{a}^{x} e^{-\lambda\left[\left(\frac{u}{a}\right)^{\theta}-1\right]} d u .
$$

which is (25).

Now for sufficient condition we need to prove (25) which implies (4). We consider (25) as

$$
h(x)=\frac{a^{\theta+1}}{\lambda \theta} x^{1-\theta} e^{\lambda\left[\left(\frac{x}{a}\right)^{\theta}-1\right]}-\frac{a^{\theta}}{\lambda \theta} x^{2-\theta}+\frac{a^{\theta}}{\lambda \theta} x^{1-\theta} e^{\lambda\left[\left(\frac{x}{a}\right)^{\theta}-1\right]} \int_{a}^{x} e^{-\lambda\left[\left(\frac{u}{a}\right)^{\theta}-1\right]} d u .
$$

Differentiating above equation with respect to $x$, we have

$$
h^{\prime}(x)=\left(\frac{(1-\theta)}{x}+\frac{\lambda \theta}{a^{\theta}} x^{\theta-1}\right)\left(\frac{a^{\theta+1}}{\lambda \theta} x^{1-\theta} e^{\lambda\left[\left(\frac{x}{a}\right)^{\theta}-1\right]}+\frac{a^{\theta}}{\lambda \theta} x^{1-\theta} e^{\lambda\left[\left(\frac{x}{a}\right)^{\theta}-1\right]} \int_{a}^{x} e^{-\lambda\left[\left(\frac{u}{a}\right)^{\theta}-1\right]} d u\right)-\frac{(1-\theta)}{\lambda \theta} a^{\theta} x^{1-\theta} .
$$

Thus we have,

$$
\frac{x-h^{\prime}(x)}{h(x)}=-\left(\frac{(1-\theta)}{x}+\frac{\lambda \theta}{a^{\theta}} x^{\theta-1}\right) .
$$

Hence, using the result of Lemma 6.1 from [16], we have

$$
\frac{g^{\prime}(x)}{g(x)}=-\left(\frac{(1-\theta)}{x}+\frac{\lambda \theta}{a^{\theta}} x^{\theta-1}\right) .
$$

On integrating, we get

$$
g(x)=c \frac{e^{-\lambda\left(\frac{x}{a}\right)^{\theta}}}{x^{1-\theta}},
$$

where $c$ is determined by using $\int_{a}^{\infty} g(x) d x=1$, hence, we get

$$
g(x)=\frac{\lambda \theta}{a^{\theta}} x^{\theta-1} e^{-\lambda\left[\left(\frac{x}{a}\right)^{\theta}-1\right]}, x>a, \theta, \lambda>0,
$$

which implies (4). 
Theorem 8

Suppose that $X$ is an absolutely continuous random variable with $\mathrm{cdf} G(x)$ such that $G(a)=0$ and $G(x)>0$ for all $x>a$. We assume that the $p d f$ of $X$ and $g(x)$ and $g^{\prime}(x)$ exists for all $x>0$ and $E(X)$ also exists. Then $E(X \mid X \geq x)=h_{0}(x) \varsigma(x)$, where $\varsigma(x)=g(x) /[1-G(x)]$,

$$
h_{0}(x)=x^{1-\theta} e^{\lambda\left[\left(\frac{x}{a}\right)^{\theta}-1\right]} \int_{x}^{\infty} u^{\theta} e^{-\lambda\left[\left(\frac{u}{a}\right)^{\theta}-1\right]} d u,
$$

holds if and only if

$$
g(x)=\frac{\lambda \theta}{a^{\theta}} x^{\theta-1} e^{-\lambda\left[\left(\frac{x}{a}\right)^{\theta}-1\right]}, \quad x \geq a, \theta, \lambda>0 .
$$

Proof

We have

$$
\begin{gathered}
g(x) h_{0}(x)=\frac{\lambda \theta}{a^{\theta}} \int_{x}^{\infty} u^{\theta} e^{-\lambda\left[\left(\frac{u}{a}\right)^{\theta}-1\right]} d u \\
h_{0}(x)=x^{1-\theta} e^{\lambda\left[\left(\frac{x}{a}\right)^{\theta}-1\right]} \int_{x}^{\infty} u^{\theta} e^{-\lambda\left[\left(\frac{u}{a}\right)^{\theta}-1\right]} d u .
\end{gathered}
$$

By differentiating the above expression, we have

$$
\begin{gathered}
h_{0}^{\prime}(x)=x+\left(\frac{(1-\theta)}{x}+\frac{\lambda \theta}{a^{\theta}} x^{\theta-1}\right) x^{1-\theta} e^{\lambda\left[\left(\frac{x}{a}\right)^{\theta}-1\right]} \int_{x}^{\infty} u^{\theta} e^{-\lambda\left[\left(\frac{u}{a}\right)^{\theta}-1\right]} d u \\
h_{0}^{\prime}(x)=x+\left(\frac{(1-\theta)}{x}+\frac{\lambda \theta}{a^{\theta}} x^{\theta-1}\right) h_{0}(x) .
\end{gathered}
$$

Using result of Lemma 6.2 from [16], we have

$$
\begin{gathered}
-\frac{x-h_{0}^{\prime}(x)}{h_{0}(x)}=-\left(\frac{(1-\theta)}{x}+\frac{\lambda \theta}{a^{\theta}} x^{\theta-1}\right) \\
\frac{g^{\prime}(x)}{g(x)}=-\frac{(1-\theta)}{x}-\frac{\lambda \theta}{a^{\theta}} x^{\theta-1} .
\end{gathered}
$$

Integrating both sides with respect to $x$, we have

$$
g(x)=c x^{\theta-1} e^{-\lambda\left(\frac{x}{a}\right)^{\theta}},
$$

where $c$ is a constant determined by using the condition $\int_{a}^{\infty} g(x) d x=1$, hence, we get

$$
g(x)=\frac{\lambda \theta}{a^{\theta}} x^{\theta-1} e^{-\lambda\left[\left(\frac{x}{a}\right)^{\theta}-1\right]}, x \geq a, \theta, \lambda>0,
$$

which implies (4).

\section{Conclusion}

In this paper, exact form for single moments from OGEPD has been established in conjunction with derivations for some recurrence relations for single, inverse, product and ratio moments. Further, first four moments of gos and upper record values from the said distribution have been calculated. A characterization by two methods is also given.

\section{REFERENCES}


1. M. Ahsanullah, and M. Shakil, A note on a characterization of J-Shaped distribution by truncated moment, Applied Mathematical Sciences, vol. 8, no. 117, pp. 5801-5812, 2014.

2. H. Athar, and H. M. U., Islam, Recurrence relations for single and product moments of generalized order statistics from a general class of distribution, Metron-International Journal of Statistics, vol.62, no. 3, pp. 327-337, 2004.

3. H. Athar, R. U. Khan, and Z. Anwar, Exact moments of lower generalized order statistics from power function distribution, Calcutta Statistical Association Bulletin, vol. 62, no. 1-2, pp. 31-46, 2010.

4. M. Faizan and H. Athar, Moments of generalized order statistics from a general class of distributions, Journal of Statistics, vol. 15, pp. 36-43, 2008

5. I. S. Gradshteyn, and I. M., Ryzhik, Table of integrals, series, and products, Academic press, 2014.

6. N. Gupta, Z. Anwar and A. A. Dar, Moment Properties of Generalized Order Statistics from Ailamujia Distribution, International Journal of Computational and Theoretical Statistics, 5(02), pp. 115-122, 2018.

7. J. S. Hwang, and G. D. Lin, Extensions of Müntz-Szász theorem and applications, Analysis, vol. 4(1-2), pp. 143-160, 1984.

8. U. Kamps, A concept of generalized order statistics, Journal of Statistical Planning and Inference, vol. 48, no. 1 pp. 1-23, 1995.

9. U. Kamps nad U. Gather, Characteristic properties of generalized order statistics from exponential distributions, Applicationes Mathematicae, vol. 24, pp. 383-391, 1997.

10. M. Arshad and Q. A. Jamal, Interval Estimation for Topp-Leone Generated Family of Distributions based on Dual Generalized Order Statistics, American Journal of Mathematical and Management Sciences:https://doi.org/10.1080/01966324.2018.1509033.

11. A. H. Khan, Z. Anwar, and H. Athar, Exact moments of generalized and dual generalized order statistics from a general form of distributions, J. Statist. Science, vol. 1, no. 1 pp. 27-44, 2009.

12. R. U. Khan, Z. Anwar, and H. Athar, Recurrence relations for single and product moments of generalized order statistics from doubly truncated Weibull distribution, Aligarh Journal of Statistics, vol.27, pp. 69-79, 2007.

13. R. U. Khan, M.A. Khan and M.A.R. Khan, Relations for moments of generalized record values from additive Weibull distribution and associated inference, Statistics, Optimization and Information Computing, 5(2), 127-136.

14. D. Kumar, and S. Dey, Relations for Moments of Generalized Order Statistics from Extended Exponential Distribution, American Journal of Mathematical and Management Sciences, vol. 36, no. 4 pp. 378-400, 2017.

15. S. S. Maiti, and S. Pramanik, Odds Generalized Exponential-Pareto Distribution: Properties and Application, Pakistan Journal of Statistics and Operation Research,vol. 12, no. 2 pp. 17-31, 2016.

16. S. H. Shahbaz, J. S. Arabia, M. Ahsanullah, and M. Q. Shahabaz, (2017) Recurrence Relations for Moments of Generalized Order Statistics for Inverse Weibull Distribution and Some Characterizations, Journal of Statistical Theory and Applications, vol. 16, no. 2, pp. 137-149, 2017. 